\title{
Boas práticas e qualidade sanitária dos alimentos servidos em restaurantes do tipo self-service no Campus da Universidade Federal do Pará
}

\author{
Lidinei do Socorro da Costa Carvalho ${ }^{1}$, Maria do Socorro Souza Ribeiro ${ }^{1}$, Consuelo Lima \\ Sousa $^{2}$ e Victor Hugo Alves do Nascimento ${ }^{3}$
}

O presente estudo teve como objetivo avaliar as condições higiênico-sanitárias de três restaurantes self-service do Campus da Universidade Federal do Pará. Para a avaliação das Boas Práticas, utilizou-se a Ficha de Verificação baseado na Resolução RDC no 216 da ANVISA. A temperatura dos alimentos foi medida com auxílio de um termômetro Infravermelho a laser e paralelamente foram coletadas 45 amostras de alimentos prontos para o consumo, sendo 15 amostras por restaurante, nas quais foram realizadas análises de coliformes a $45^{\circ} \mathrm{C}$, Salmonella spp, estafilococos coagulase positiva e clostrídios sulfito redutores a $46^{\circ} \mathrm{C}$, obedecendo a metodologia descrita no Compendium of Methods for the Microbiological Examination of Foods. Os restaurantes A e B obtiveram em média 61,4\% e 62,3\% de conformidade, respectivamente, qualificando-se como Regular. O restaurante C com média de 89,3\% de conformidades foi classificado como Bom. A maioria das amostras (91\%) apresentou temperatura inadequada. Não foi detectada contaminação por Salmonella spp., estafilococos coagulase positiva e clostrídios sulfito redutores a $46^{\circ} \mathrm{C}$. Para a pesquisa de coliformes, $57,8 \%$ das amostras apresentaram contaminação acima do limite preconizado pela legislação vigente. Conclui-se que há necessidade de adequação dos restaurantes às exigências da legislação.

Palavras-chave: Lista de Verificação; Segurança do Alimento; Contaminação; Higiene.

\section{Good practices and sanitary quality of food served in self-service restaurants at Federal University of Pará}

This study aimed to evaluate the sanitary conditions of three self-service restaurants at the campus of the Federal University of Pará. For that, a check list based on RDC Resolution 216 was used. The food temperature was measured using a laser infrared thermometer. Forty-five samples of food ready for consumption were collected, 15 samples per restaurant, for enumeration of Coliforms at $45^{\circ} \mathrm{C}$, Salmonella spp, coagulase-positive staphylococci and clostridium sulphite reducers $46^{\circ} \mathrm{C}$, based on the methodology described in the Compendium of Methods for the Microbiological Examination of Foods. The restaurants A and B obtained an average $61.4 \%$ and $62.3 \%$ of compliance, respectively, thus being classified as regular. The restaurant $\mathrm{C}$ with an average of $89.3 \%$ of compliance was rated as good. Most of the samples (91\%) had inadequate temperature. Contamination by Salmonella spp.,

\footnotetext{
${ }^{1}$ Discente do Curso de Engenharia de Alimentos da Universidade Federal do Pará.

${ }^{2}$ Professora Doutora da Universidade Federal do Pará, Instituto de Tecnologia, Faculdade de Engenharia de Alimentos. Endereço para correspondência: Rua Augusto Corrêa no 1, Guamá, CEP: 60075-110, Belém, Pará. Telefone: +55 (91) 9812-97760. Fax: +55 (91) 3201-8055. E-mail: sousa@ufpa.br

${ }^{3}$ Discente no Programa de Pós-Graduação em Ciência e Tecnologia de Alimentos da Universidade Federal do Pará.
} 
Coagulase-positive staphylococci and clostridium sulphite reducers $46^{\circ} \mathrm{C}$ were not detected, while $57.8 \%$ of the samples presented contamination by coliform above the recommended limit by law. In conclusion, it is necessary to adapt the restaurants to the requirements of the legislation.

Keywords: Checklist; Food Safety; Contamination; Hygiene.

\section{INTRODUÇÃO}

Em virtude das intensas mudanças ocorridas nas últimas décadas, transformações que afetaram o estilo de vida da população, tem-se verificado um aumento significativo da alimentação fora do domicílio [1]. As dificuldades impostas pelos longos deslocamentos e a extensa jornada de trabalho impedem que a maioria dos indivíduos realize suas refeições em família [2].

O restaurante de comida a quilo (self-service) mostrou-se uma excelente opção para quem trabalha fora e quer pagar relativamente pouco, comendo bem e em pouco tempo. No entanto, as condições de higiene em que esses alimentos são preparados e a temperatura a que ficam submetidos durante a exposição nem sempre são ideais, podendo desenvolver microorganismos potencialmente perigosos para a saúde do consumidor ${ }^{[3]}$.

Existe um grande número de fatores que contribuem para tornar um alimento inseguro, que causa doenças microbianas de origem alimentar às pessoas que ingerem. Entre as principais causas estão o controle inadequado da temperatura durante $\mathrm{O}$ cozimento, o resfriamento e a estocagem, higiene pessoal insuficiente, contaminação cruzada entre produtos crus e processados, e monitoramento inadequado dos processos [4]. Entre as bactérias patogênicas, destacam-se na maioria das infecções e intoxicações alimentares: Listeria monocytogenes, Salmonella spp., Escherichia coli, Clostridium Sulfito Redutor e Staphylococcus aureus, pois estão entre os principais microorganismos que causam preocupação em relação a carnes cruas e processadas e a ambientes de manipulação de alimentos [5].

Em ambientes universitários circulam diariamente milhares de pessoas (alunos, funcionários, professores e visitantes), todos potenciais consumidores de alimentos nas diversas cantinas e restaurantes ${ }^{[6]}$. A elaboração das refeições nestes estabelecimentos, no que diz respeito à qualidade higiênico-sanitária, deve ocorrer de forma a garantir a produção do alimento seguro por meio de, dentre outros, da adoção das Boas Práticas de Fabricação (BPF) - procedimentos que devem ser adotados por serviços de alimentação a fim de garantir a qualidade higiênico-sanitária e a conformidade dos alimentos com a legislação sanitária [3,].

Desta forma o presente trabalho tem como objetivo avaliar as condições higiênico-sanitárias de restaurantes self-service do Campus da Universidade Federal do Pará - UFPA, dentro do que preconiza a legislação brasileira sobre Boas Práticas de Fabricação, visando a produção de alimentos seguros.

\section{MATERIAL E MÉTODOS}

O trabalho foi realizado em três restaurantes self-service localizados no Campus da Universidade Federal do Pará em Belém (Pará), denominados por A, B e C.

\section{Avaliação das Boas Práticas}

Para a avaliação das Boas Práticas utilizou-se a Ficha de Verificação das Boas Práticas (FVBP) para Serviços de Alimentação baseado na Resolução RDC no 216/2004 da Agência Nacional de Vigilância Sanitária (ANVISA) [8], composta de 12 blocos com 114 itens: Bloco 1 - Edificação, instalações, móveis e utensilios (27 itens); Bloco 2 - Higienização de instalações, equipamentos, móveis e utensilios (11 itens); Bloco 3 - Controle integrado de vetores e pragas urbanas (1item); Bloco 4 - Abastecimento de água (5 itens); Bloco 5 - Manejo dos resíduos (6 itens); Bloco 6 - Manipuladores (12itens); Bloco 7 - Matérias-primas, ingredientes e embalagens (9 itens); Bloco 8 Preparação do alimento (13 itens); Bloco 9 Armazenamento e transporte do alimento preparado (3 itens); Bloco 10 - Exposição ao consumo do alimento preparado (8 itens); Bloco 11 - Documentação e registro (17 itens); e Bloco 12 - Responsabilidade (2 itens). 
Os itens foram classificados de acordo com os seguintes critérios: Conforme (C) quando estavam em conformidade com a legislação sanitária vigente; Não Conforme (NC) quando não estavam em conformidade e Não se Aplica (NA) para os itens que não tinham relevância. Cada bloco da ficha de avaliação obteve um valor, em porcentagem, em função da conformidade ou não conformidade dos itens. Todos os itens atendidos foram somados para obter a porcentagem geral do estabelecimento. A classificação geral do estabelecimento foi feita de acordo com o estabelecido na legislação [8]: Grupo 1 - BOM (76 a $100 \%$ de atendimento dos itens), Grupo 2 REGULAR (51 a $75 \%$ de atendimento dos itens) e Grupo 3 - RUIM ( 0 a $50 \%$ de atendimento dos itens).

Para o preenchimento dos itens da FVBP foram realizadas três vistorias em cada estabelecimento, em setembro de 2015 (1 1 a Vistoria), dezembro de 2015 (2a Vistoria) e em fevereiro de 2016 (3a Vistoria), nas quais se acompanhou a rotina de trabalho de cada restaurante. Após cada vistoria e a análise dos resultados, foi entregue aos proprietários dos restaurantes o plano de ação com as não conformidades e suas respectivas ações corretivas para melhoria da qualidade dos serviços.

\section{Avaliação das temperaturas}

Durante as visitas aos restaurantes para a coleta das amostras de alimentos para análise, verificou-se a temperatura durante a exposição nos balcões de distribuição com auxílio de um Termômetro Infravermelho a Laser Mini Temp MT4 Raytek ${ }^{\circledR}$ com faixa de temperatura entre $-18^{\circ} \mathrm{C}$ a $+400^{\circ} \mathrm{C}$. Foram aferidas as temperaturas de cada alimento 3 vezes e o resultado expresso considerou a média de três medições.

\section{Análises microbiológicas}

Foram coletadas um total de 45 amostras diretamente dos balcões de distribuição (15 amostras por restaurante), assim distribuídas: 5 de pratos quentes a base de carnes, 5 de salada crua, e 5 de suco de fruta.

As amostras foram transportadas até $\mathrm{O}$ laboratório em recipientes de vidro estéreis, devidamente identificados e acondicionados em caixas de material isotérmico. Em seguida foram analisadas quanto aos parâmetros exigidos pela Legislação vigente
- RDC no 12 [9] para cada tipo de alimento, sendo: suco e salada crua: contagem de coliformes a $45^{\circ} \mathrm{C}$ e pesquisa de Salmonella spp/25g; proteína (pratos prontos para consumo à base de carnes): contagem de coliformes a $45^{\circ} \mathrm{C}$, clostrídios sulfito redutor a $46^{\circ} \mathrm{C}$, estafilococus coagulase positiva/g e pesquisa de Salmonella spp/25g. Todas as análises seguiram metodologias descritas no Compendium of Methods for the Microbiological Examination of Foods [10].

\section{RESULTADOS E DISCUSSÃO}

\section{Avaliação das Boas Práticas}

A Figura 1 mostra a porcentagem geral de conformidades, por vistoria, de cada restaurante. Os restaurantes A e B obtiveram em média 61,4\% e 62,3\% de conformidade, respectivamente, desta forma estão enquadrados no Grupo 2 com classificação "Regular". Entretanto, observa-se, no decorrer das vistorias melhoria do nível de conformidades nos dois estabelecimentos avaliados (Figura 1). O restaurante $\mathrm{C}$ manteve-se durantes as três vistorias dentro do intervalo (Grupo 1) e com média de 89,3\% de conformidades e classificado como "Bom", sendo este o único com responsável técnico com formação superior (nutricionista). É recomendado para os serviços de alimentação que os atributos avaliados tenham porcentagens de conformidades maiores que $70,0 \%$ para garantir a produção de alimentos seguros [11].

Figura 1. Percentual de conformidades em cada vistoria* dos restaurantes do tipo self-service do Campus da UFPA Belém

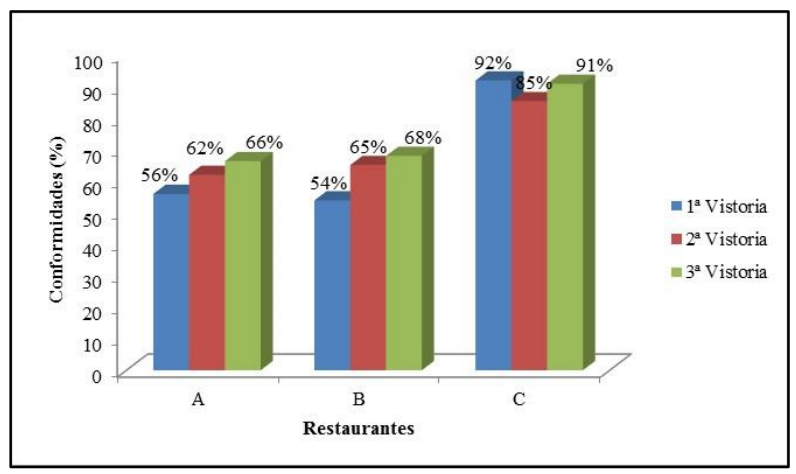

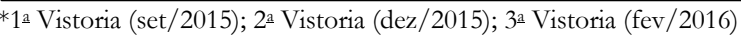


Ao realizar avaliação das condições higiênicosanitárias em 17 restaurantes tipo self-service do município de Patos de Minas, Rocha et al. [12], observaram que $23,5 \%$ dos restaurantes tem baixa adequação $(0-50 \%$ de atendimento dos itens), 70,6\% têm média adequação $(51-75 \%$ de atendimento dos itens) e somente 1 restaurante $(5,9 \%)$ tem alta adequação $(76-100 \%$ de atendimento dos itens), sendo também o único que possui nutricionista no seu quadro de funcionários.

A maior adequação à Ficha de Verificação está diretamente ligada à presença de um técnico responsável com formação voltada para manipulação de alimentos e boas práticas, pois estes são aptos a fazer correta orientação e supervisão das atividades, capacitação dos funcionários e gerenciamento das etapas que envolvem a produção segura dos alimentos [13].

Analisando a Tabela 1, observa-se que todos obtiveram 100\% de conformidade nos Blocos 3, 4 e 12, controle integrado de vetores e pragas urbanas, abastecimento de água e responsabilidades, respectivamente, em contrapartida $100 \%$ de não conformidade no Bloco 9 (armazenamento e transporte do alimento preparado), pois em nenhum dos restaurantes constatou-se identificação com data de preparo e prazo de validade dos alimentos preparados.

Tabela 1. Percentual de conformidade de cada Bloco da FVBP em cada vistoria dos restaurantes tipos self-service do Campus da UFPA - Belém

\begin{tabular}{|c|c|c|c|c|c|c|c|c|c|}
\hline \multirow{2}{*}{ BLOCO } & \multicolumn{3}{|c|}{$\begin{array}{c}\text { Restaurante A } \\
(\%)\end{array}$} & \multicolumn{3}{|c|}{$\begin{array}{c}\text { Restaurante B } \\
(\%)\end{array}$} & \multicolumn{3}{|c|}{$\begin{array}{c}\text { Restaurante C } \\
(\%)\end{array}$} \\
\hline & $1 \underline{\mathrm{a} V}$ & $2 \mathrm{a} V$ & $3 \mathrm{a} \mathrm{V}$ & $1 \underline{a} \mathrm{~V}$ & $2 \mathrm{a} V$ & $3 \mathrm{a} V$ & $1 \underline{a} \mathrm{~V}$ & $2 \mathrm{a} V$ & $3 \mathrm{a} V$ \\
\hline 1 & 58 & 63 & 67 & 52 & 64 & 72 & 85 & 81 & 85 \\
\hline 2 & 80 & 80 & 90 & 80 & 90 & 90 & 100 & 100 & 100 \\
\hline 3 & 100 & 100 & 100 & 100 & 100 & 100 & 100 & 100 & 100 \\
\hline 4 & 100 & 100 & 100 & 100 & 100 & 100 & 100 & 100 & 100 \\
\hline 5 & 83 & 100 & 100 & 83 & 100 & 100 & 83 & 83 & 83 \\
\hline 6 & 50 & 70 & 80 & 36 & 73 & 73 & 92 & 75 & 92 \\
\hline 7 & 100 & 100 & 100 & 75 & 89 & 89 & 78 & 78 & 89 \\
\hline 8 & 50 & 57 & 71 & 62 & 73 & 82 & 100 & 73 & 82 \\
\hline 9 & 0 & 0 & 0 & 0 & 0 & 0 & 0 & 0 & 0 \\
\hline 10 & 63 & 75 & 75 & 63 & 63 & 63 & 100 & 88 & 100 \\
\hline 11 & 0 & 0 & 0 & 0 & 0 & 0 & 100 & 100 & 100 \\
\hline 12 & 100 & 100 & 100 & 100 & 100 & 100 & 100 & 100 & 100 \\
\hline
\end{tabular}

Bloco 1 - Edificação, instalações, móveis e utensílios; Bloco 2 - Higienização de instalações, equipamentos, móveis e utensílios; Bloco 3 - Controle integrado de vetores e pragas urbanas; Bloco 4 - Abastecimento de água; Bloco 5 - Manejo dos resíduos; Bloco 6 - Manipuladores; Bloco 7 - Matérias-primas, ingredientes e embalagens; Bloco 8 - Preparacão do alimento; Bloco 9 Armazenamento e transporte do alimento preparado; Bloco 10 - Exposição ao consumo do alimento preparado; Bloco 11 - Documentação e registro; Bloco 12 - Responsabilidade.

$1 \mathrm{a} \mathrm{V}=1 \mathrm{a}$ Vistoria (set/2015); 2a $\mathrm{V}=2 \mathrm{a}$ Vistoria (dez/2015); 3a $\mathrm{V}=3 \mathrm{a}$ Vistoria (fev/2016).

Em relação à edificação, instalações e utensilios (Bloco 1), constatou-se que em todos os restaurantes não há possibilidade de um fluxo ordenado e sem cruzamentos em todas as etapas da preparação de alimentos; as instalações não possuem acesso controlado e independente; e o dimensionamento da edificação e das instalações não é compatível com todas as operações. Isto se deve ao fato de que os restaurantes estão localizados em espaços improvisados, o que acaba impedindo uma estruturação adequada para manipulação e comercialização de alimentos. As inadequações apontadas resultam tanto de falhas da própria instituição, quanto do próprio estabelecimento responsável pela manutenção da área física interna ${ }^{[}{ }^{6}$. 
Ao avaliarem a adequação de restaurantes comerciais às Boas Práticas, Santos et al. ${ }^{[14]}$ também verificaram espaços físicos não projetados para tal finalidade e expandidos inadequadamente, impossibilitando um fluxo ordenado das etapas de preparação e distribuição dos alimentos.

As "não conformidades" mais comuns Bloco 5 (manejo de resíduos) foram a falta de identificação nos recipientes de coleta de lixo e coletores com acionamento manual. Recipientes sem acionamento automático podem contribuir para o aumento do risco de contaminação de alimentos. $\mathrm{Na}$ maioria das vezes a não aquisição das mesmas está relacionada à falta de conhecimento dos responsáveis sobre a importância da exigência legal [15]. O manejo de resíduos inadequado pode gerar contaminação cruzada ou atrair vetores $[14,15]$.

No Bloco 6 (manipuladores) a porcentagem de conformidades variou de $36,4 \%$ a $91,7 \%$, sendo a utilização de adornos pelos funcionários um problema encontrado em todos os restaurantes, e a falta de sanificante para higienização das mãos um ponto crítico para segurança. Messias et al. ${ }^{[16]}$ consideram a lavagem das mãos como um ponto crítico de controle nos serviços de alimentação, tornando-se necessária a implantação de procedimentos padronizados de lavagem e a fixação de cartazes sobre a correta lavagem das mãos nas cozinhas de serviços de alimentação.

No que diz respeito à documentação e registros (Bloco 11), os restaurantes $\mathrm{A}$ e $\mathrm{B}$ não apresentaram conformidade durante as três vistorias, pois estes ainda não dispõem de Manual de Boas Práticas e nem de Procedimentos Operacionais Padronizados. Em contrapartida, o restaurante C atendeu a todos os itens do Bloco 11, mantendo-se com $100,0 \%$ de conformidades durante as vistorias. Os serviços de alimentação como padarias, lanchonetes, cozinhas industriais, cozinhas institucionais, rotisserias e restaurantes devem possuir, obrigatoriamente, o manual de Boas Práticas de Fabricação para aplicação das normas em suas unidades, a fim de garantir a segurança do alimento ${ }^{[8]}$.
Em estudo realizado por São José et al. [17], observaram-se 75,0\% de adequação ao avaliar as boas práticas em uma unidade de alimentação e nutrição no município de Contagem (Minas Gerais), enquadrandose no Grupo 2 (Regular). Os autores evidenciam que o maior percentual de adequação foi verificado no bloco relativo aos manipuladores e o maior percentual de inadequação, no bloco que envolvem a documentação.

\section{Avaliação das temperaturas}

A Tabela 2 apresenta as médias das temperaturas das amostras de suco, salada e proteína (produto pronto para consumo a base de carnes) coletadas. De acordo com legislação ${ }^{[8]}$, as preparações quentes no momento da distribuição devem ser mantidas em temperatura $\geq 60^{\circ} \mathrm{C}$, e as preparações frias até $5^{\circ} \mathrm{C}$ para garantir a segurança do produto. Temperaturas inadequadas colocam as preparações em risco, pois possibilitam a multiplicação dos microorganismos e assim podem ocasionar $\mathrm{O}$ desenvolvimento de doenças veiculadas por alimentos [18]. 
Tabela 2. Resultado das temperaturas das amostras de alimentos quentes e frios coletadas nos restaurantes self-service da UFPA - Belém

\begin{tabular}{cccccc}
\hline \multirow{2}{*}{ Restaurante } & Amostra & $\mathrm{n}$ & \multicolumn{3}{c}{ Temperatura $\left({ }^{\circ} \mathrm{C}\right)$} \\
\cline { 4 - 6 } & & & Média \pm DP & Mínimo & Máximo \\
\hline \multirow{2}{*}{ A Suco } & 5 & $10,0 \pm 4,1$ & 4,3 & 14,7 \\
& Salada & 5 & $24,8 \pm 2,3$ & 22,2 & 27,7 \\
& Proteína & 5 & $46,7 \pm 3,8$ & 41,5 & 50,8 \\
\hline \multirow{2}{*}{ B } & Suco & 5 & $5,1 \pm 2,8$ & 2,8 & 9,3 \\
& Salada & 5 & $20,5 \pm 1,1$ & 19,3 & 21,8 \\
& Proteína & 5 & $41,2 \pm 4,2$ & 35,3 & 46,7 \\
\hline & Suco & 5 & $18,0 \pm 6,3$ & 11,5 & 28,3 \\
& Salada & 5 & $27,6 \pm 2,2$ & 24,5 & 30,2 \\
& Proteína & 5 & $49,4 \pm 5,3$ & 45,5 & 58,8 \\
\hline
\end{tabular}

$\mathrm{n}=$ número de amostras; $\mathrm{DP}=$ desvio padrão

Das 45 amostras coletadas 91,1\% (41/45) apresentavam temperatura fora do recomendado para manter a segurança do alimento. As médias das temperaturas das preparações quentes variaram de $35,3^{\circ} \mathrm{C}$ a $58,8^{\circ} \mathrm{C}$, estando todas inadequadas para este grupo de alimentos. Nas preparações frias a variação foi de $2,8^{\circ} \mathrm{C}$ a $30,2^{\circ} \mathrm{C}$, neste grupo $100,0 \%(15 / 15)$ das amostras de salada crua estavam em desacordo com o recomendado, e apenas $26,7 \%$ (4/15) das amostras de suco encontravam-se em temperatura de até $5^{\circ} \mathrm{C}$.

Resultados similares foram encontrados por Rocha et al. ${ }^{[12]}$, que observaram que 100,0\% (71/71) das amostras de salada crua estavam em temperatura considerada insegura. Ao avaliar as temperaturas nas preparações quentes e frias dos restaurantes self-service do hipercentro de Belo Horizonte/MG, Oliveira et al. [18] observaram a variação de $36,8^{\circ} \mathrm{C}$ a $45,8^{\circ} \mathrm{C}$ em preparações quentes, e $19,08^{\circ} \mathrm{C}$ a $20,58^{\circ} \mathrm{C}$ em preparações frias, mostrando que todas as amostras analisadas estavam com temperaturas inadequadas.

Em outro estudo Alves e Ueno [19], observaram um intervalo de $33^{\circ} \mathrm{C}$ a $71,2^{\circ} \mathrm{C}$ para alimentos quentes, sendo que apenas 22,0\% (7/32) estavam em temperatura que confere segurança aos consumidores, e $3,1 \%(1 / 32)$ dos alimentos frios encontrava-se em condições adequadas, com variação de temperatura entre $9,7^{\circ} \mathrm{C}$ e $41,7^{\circ} \mathrm{C}$. Penedo et al. [20], quando avaliaram as temperaturas dos alimentos durante o preparo e distribuição em restaurantes comerciais de Belo Horizonte-MG, observaram que apenas 40,0\% (4/10) apresentaram temperaturas adequadas das preparações quentes, em relação às preparações frias, todas se mantiveram em temperaturas superiores a $10^{\circ} \mathrm{C}$.

\section{Análises microbiológicas}

Em nenhuma das amostras analisadas (suco, salada crua e proteína) foi detectada a presença de Salmonella spp., e a contagem de Estafilococus coagulase positiva e de Clostridium sulfito redutor a $46^{\circ} \mathrm{C}$ foi $<1,0 \times 101 \mathrm{UFC} / \mathrm{g}$ em todas as amostras, atendendo a legislação brasileira [9]. Estes resultados demonstram que as preparações analisadas não oferecem risco direto à saúde do consumidor, visto que a ocorrência desses micro-organismos patogênicos constitui sério problema para a saúde pública, uma vez que causam graves doenças no homem [5].

Com relação à avaliação de coliformes a $45^{\circ} \mathrm{C}$ (Tabela 3), observa-se que 46,7\% (7/15), 86,7\% (13/15) e 40,0\% (6/15) das amostras de suco, salada crua e proteína, respectivamente, apresentaram contaminação acima do limite preconizado pela legislação vigente [?]. Coliformes a $45^{\circ} \mathrm{C}$ compreende um grupo de bactérias indicadoras de contaminação utilizadas na avaliação da 
qualidade higiênico-sanitária de alimentos prontos para consumo [21].

Das amostras de suco com contaminação acima do permitido pela legislação vigente, $60,0 \%(3 / 5)$ foram coletadas no restaurante A, e 80,0\% (4/5) no restaurante $C$. Todas as amostras coletadas no restaurante $B$ apresentaram contagem $<3,0 \mathrm{NMP} / \mathrm{mL}$. Este resultado pode ser associado à qualidade da matéria-prima, às condições adequadas de higiene, e às boas condições de conservação do produto, visto que no momento da coleta $60,0 \%$ (3/5) estavam em temperatura adequada para garantir a segurança do alimento.

Brum et al. [22], ao avaliarem a qualidade microbiológica de refrescos comercializados nos municípios de Barra Mansa e Volta Redonda - RJ, verificaram $29,0 \%$ de amostras da bebida em desacordo com os padrões estabelecidos pela legislação vigente quanto à presença de coliformes a $45^{\circ} \mathrm{C}$. Silveira et al. [23] avaliando sucos de laranja in natura encontraram resultados na faixa de $<3,0$ a $930,0 \mathrm{NMP} / \mathrm{mL}$ e do total, $33,3 \%$ das amostras apresentaram com contagem acima $100,0 \mathrm{NMP} / \mathrm{mL}$. Contagens superiores a 100,0 $\mathrm{NMP} / \mathrm{mL}$ para coliformes a $45^{\circ} \mathrm{C}$ sustentam a afirmação de que os sucos foram submetidos a condições inadequadas de manipulação e/ou estocagem [24].

Tabela 3. Resultado da contagem de coliformes a $45^{\circ} \mathrm{C}$ nas amostras de alimentos coletadas nos restaurantes do tipo self-service do Campus da UFPA - Belém

\begin{tabular}{cccc}
\hline NMP $/ \mathrm{g}$ & $\begin{array}{c}\text { Suco } \\
\mathrm{n}\end{array}$ & $\begin{array}{c}\text { Salada Crua } \\
\mathrm{n}\end{array}$ & $\begin{array}{c}\text { Proteína } \\
\mathrm{n}\end{array}$ \\
\hline$<3$ & 6 & 1 & 8 \\
$3-10$ & 1 & - & 1 \\
$>10-20$ & - & - & - \\
$>20-100$ & 1 & 1 & 3 \\
$>100-1.000$ & 3 & 3 & 1 \\
$\geq 1.100$ & 4 & 10 & 2 \\
\hline BRASIL [9] & $10^{2} \mathrm{NMP} / \mathrm{ml}$ & $10^{2} \mathrm{NMP} / \mathrm{g}$ & $2 \times 10 \mathrm{NMP} / \mathrm{g}$ \\
\hline $\mathrm{n}=$ número de amostras & & &
\end{tabular}

As amostras de salada crua inadequadas para o consumo devido a elevada contaminação por coliformes a $45^{\circ} \mathrm{C}\left(>10^{2} \mathrm{NMP} / \mathrm{g}\right)$, representam $80,0 \%$ (4/5) das amostras coletadas no restaurante A, 80,0\%
(4/5) no restaurante $\mathrm{B}$ e $100,0 \%$ (5/5) no restaurante $\mathrm{C}$. A presença de coliformes a $45^{\circ} \mathrm{C}$ demonstra falhas no processo de higienização das hortaliças ou contaminação cruzada, no qual os utensilios e superfícies entram em contato com o alimento durante o seu preparo [25].

Junior et al. [26] ao traçarem o perfil parasitológico e microbiológico de alfaces comercializadas em restaurantes self-service de Gurupi (Tocantins), observaram 68,4\% (13/19) de amostras em desacordo com os padrões de coliformes termotolerantes exigidos pela legislação, ressaltando que a qualidade destas amostras esteve próxima a de um produto sem tratamento, oferecendo risco potencial ao consumidor quanto à presença de patógenos entéricos. A contaminação por coliformes a $45^{\circ} \mathrm{C}$ em saladas cruas é também relatada por outros autores ${ }^{[27,28]}$.

As amostras de proteínas (pratos prontos para consumo a base de carnes) com contagens elevadas de coliformes a $45^{\circ} \mathrm{C} \quad(>2,0 \times 101 \quad \mathrm{NMP} / \mathrm{g})$ foram detectadas em $20,0 \%$ (1/5), 40,0\% (2/5) e 40,0\% (2/5) das amostras coletadas no restaurante $\mathrm{A}, \mathrm{B}$ e C, respectivamente. Os coliformes isolados nos alimentos, além de caracterizarem produtos impróprios para o consumo podem provocar surtos de infecção alimentar [29].

\section{CONCLUSÕES}

Os restaurantes A e B obtiveram em média $61,4 \%$ e $62,3 \%$ de conformidade, respectivamente, qualificando-se como Regular. O restaurante C com média de 89,3\% de conformidades foi classificado como Bom. Considerando que as Boas Práticas são as condições de organização e higiene necessárias para garantir a produção de alimentos seguros, conclui-se que há necessidade de adequação dos restaurantes às exigências da legislação, principalmente a respeito da estruturação física, a fim de melhorar a qualidade dos alimentos comercializados e proteger a saúde do consumidor.

A maioria das amostras (91\%) apresentou temperatura inadequada, porém não foi detectada contaminação por Salmonella spp., estafilococos coagulase positiva e clostrídios sulfito redutores a $46^{\circ} \mathrm{C}$. A impropriedade ao consumo de $57,8 \%$ das amostras analisadas, pelo motivo da elevada contaminação por coliformes a $45^{\circ} \mathrm{C}$, conota a necessidade de adoção das 
condições higiênico-sanitárias adequadas, além da garantia da qualidade da matéria-prima adquirida, e cuidados durante a manipulação evitando o risco de contaminação cruzada.

\section{REFERÊNCIAS}

[1] Leal D. Crescimento da alimentação fora do domicílio. Segur. Aliment. Nutr. 2010;17(1):123-32.

[2] Cardoso RCV, Souza EVA, Santos PQ. Unidades de alimentação e nutrição nos campi da Universidade Federal da Bahia: um estudo sob a perspectiva do alimento seguro. Rev. Nutr. 2005;18(5):669-80.

[3] Peixoto Joele MRS, Sousa CL, Lourenco LFH. Serviços de alimentação comercial: fator de risco para a saúde pública. Rev. Inst. Adolfo Lutz. 2014;73(1):112-17.

[4] Oliveira ABA, Paula CMD, Capalonga R, Cardoso, MRI, Tondo EC. Doenças Transmitidas por Alimentos: Principais Agentes Etiológicos, Alimentos Envolvidos e Fatores Predisponentes. Rev. HCPA. 2010;30(3):279-85.

[5] Machado JR, Marson JM, Oliveira ACS, Silva PR, Terra APS. Avaliação microbiológica das mãos $\mathrm{e}$ fossas nasais de manipuladores de alimentos da unidade de alimentação e nutrição de um hospital universitário. Medicina. 2009;42(4):46165.

[6] Arbos KA, Martins AMA, Almeida IKC, Oliveira PML, Farias LRG. Avaliação diagnóstica das condições higiênicosanitárias das cantinas em campus universitário público, João Pessoa/PB, Brasil. Rev. Contexto Saúde. 2015;15(28):84-94.

[7] Cardoso RCV, Souza EVA, Santos PQ. Unidades de alimentação e nutrição nos campi da Universidade Federal da Bahia: um estudo sob a perspectiva do alimento seguro. Rev. Nutr. 2005;18(5):669-80.

[8] Brasil. Ministério da Saúde. Agência Nacional de Vigilância Sanitária. Resolução - RDC no 216, de 15 de setembro de 2004. Dispõe sobre o Regulamento Técnico de Boas Práticas para Serviços de Alimentação. Diário Oficial da União. 16 set 2004.

[9] Brasil. Ministério da Saúde. Agência Nacional de Vigilância Sanitária. Resolução - RDC no 12, de 2 de janeiro de 2001. Regulamento técnico sobre padrões microbiológicos para alimentos. Diário Oficial da União. 10 jan 2001.

[10] Downes FP, Ito K. Compendium of methods for the microbiological. Examinations of Foods. 4. ed. Washington, DC: APHA; 2001.
[11] Sousa CL, Neves ECA, Lourenço LFH, Costa EB, Monteiro RRC. Diagnóstico das condições higiênico-sanitárias e microbiológicas de empresa fornecedora de comidas congeladas light na cidade de Belém/PA. Alim. Nutr. 2009;20(3):375-81.

[12] Rocha B, Batista LS, Borges BMA, Paiva AC. Avaliação das condições higiênico-sanitárias e da temperatura das refeições servidas em restaurantes comerciais do tipo self-service. Perquirere. 2010;7(1):30-40.

[13] Monteiro MAM, Fróes JAC, Fontes RBR, Ribeiro RC. Qualidade na produção de refeições em restaurantes do tipo selfservice. Demetra. 2014;9(4):955-61.

[14] Santos MOB, Rangel VP, Azeredo DP. Adequação de restaurantes comerciais às boas práticas. Hig. Aliment. 2010;24(190/191):44-49.

[15] Miranda ACB, Baião RCL. Avaliação das boas práticas na fabricação de preparações à base de pescados crus em restaurante japonês. C\&D-Rev. Eletr. Fainor. 2011;4(1):52-61.

[16] Messias GM, Reis MER, Soares LP, Fernandes NM, Duarte ES. Avaliação das condições higiênico-sanitárias de restaurantes do tipo self-service e do conhecimento dos manipuladores de alimentos quanto à segurança do alimento na cidade do Rio de Janeiro, RJ. Rev. Novo Enfoque. 2013;17:73-88.

[17] São José JFB, Coelho AIM, Ferreira KR. Avaliação das boas práticas em unidade de alimentação e nutrição no município de Contagem-MG. Alim. Nutr. 2011;22(3):479-87.

[18] Oliveira LC, Flores RR, Amorim MMA, Ferreira CC, Amaral DA. Avaliação das temperaturas das preparações dos restaurantes self-service do hipercentro de Belo Horizonte/MG. HU Revista. 2012;38(2):45-51.

[19] Alves MG, Ueno M. Restaurantes self-service. segurança e qualidade sanitária dos alimentos servidos. Rev. Nutr. 2010;23(4):573-80.

[20] Penedo AO, Jesus RB, Silva SCF, Monteiro MAM, Ribeiro RC. Avaliação das temperaturas dos alimentos durante o preparo e distribuição em restaurantes comerciais de Belo Horizonte MG. Demetra. 2015;10(2):429-40.

[21] Chouman K, Ponsano EHG, Michelin AF. Qualidade microbiológica de alimentos servidos em restaurantes self-service. Rev. Inst. Adolfo Lutz. 2010;69(2):261-66.

[22] Brum DCM, Mallet ACT, Saron MLG, Souza EB, Costa LMAS. Qualidade microbiológica e físico-química de refrescos comercializados nos municípios de Barra Mansa e Volta Redonda - RJ. Demetra. 2014;9(4):943-53. 
[23] Silveira MLR, Bertagnolli SMM. Avaliação microbiológica e das condições higiênico-sanitárias de comercialização de sucos de laranja in natura. Alim. Nutr. 2012;23(3):461-66.

[24] Oliveira JC, Setti-Perdigão P, Siqueira KAG, Santos AC, Miguel MAL. Características microbiológicas do suco de laranja in natura. Cienc. Tecnol. Aliment. 2006;26(2):241-45.

[25] Santos MS, Evangelista-Barreto NS, Silva RAR, Reis NA, Bernardes FS. Risco microbiológico no consumo de saladas cruas e cozidas servidas em restaurantes self-service em Cruz das Almas, Bahia, Brasil. Magistra. 2015;27(2):255-62.

[26] Junior JP, Gontijo EEL, Silva MG. Perfil parasitológico e microbiológico de alfaces comercializadas em restaurantes selfservice de Gurupi - TO. Rev. Cient. ITPAC. 2012;5(1):33-37.
[27] Von Dolinger EJO, Melo PC, Morais GR, Silva CRM, Brito $\mathrm{D}$ von D. Contaminação microbiológica de alimentos comercializados em restaurantes de auto-serviço de Itumbiara GO. Biotemas. 2010;23(4):129-33.

[28] Frittoli RB, Rodrigues LH. Análise de coliformes termotolerantes e Salmonella spp em amostras de hortaliças minimamente processadas. Rev. Cient. FHO. 2014;2(2):14-20.

[29] Passos EC, Mello ARP, Sousa CV, Silva CR, Alonso ACB, Gonzalez E, Tavares M. Provável surto de toxinfecção alimentar em funcionários de uma empresa no litoral da região sudeste do Brasil. Rev. Inst. Adolfo Lutz. 2010;69(1):136-140. 